\title{
STATL: An Attack Language for State-based Intrusion Detection
}

Steven T. Eckmann

\author{
Giovanni Vigna \\ Reliable Software Group \\ Department of Computer Science \\ University of California \\ Santa Barbara, CA 93106 \\ [ eckmann, vigna, kemm] @cs.ucsb.edu
}

Richard A. Kemmerer

\begin{abstract}
STATL is an extensible state/transition-based attack description language designed to support intrusion detection. The language allows one to describe computer penetrations as sequences of actions that an attacker performs to compromise a computer system. A STATL description of an attack scenario can be used by an intrusion detection system to analyze a stream of events and detect possible ongoing intrusions. Since intrusion detection is performed in different domains (i.e., the network or the hosts) and in different operating environments (e.g., Linux, Solaris, or Windows NT), it is useful to have an extensible language that can be easily tailored to different target environments. STATL defines domain-independent features of attack scenarios and provides constructs for extending the language to describe attacks in particular domains and environments. The STATL language has been successfully used in describing both network-based and host-based attacks, and it has been tailored to very different environments, e.g., Sun Microsystems' Solaris and Microsoft's Windows NT. An implementation of the runtime support for the STATL language has been developed and a toolset of intrusion detection systems based on STATL has been implemented. The toolset was used in a recent intrusion detection evaluation effort, delivering very favorable results. This paper presents the details of the STATL syntax and its semantics. Real examples from both the host and network-based extensions of the language are also presented.
\end{abstract}

\section{Introduction}

Intrusion detection systems analyze information about the activities performed in a computer system or network, looking for evidence of malicious behavior [1]. The information may come in the form of audit records produced by the operating system auditing facilities, log messages produced by different types of sensors, network devices, and applications, or in the form of raw network traffic obtained by eavesdropping a network segment. These data sources are used by intrusion detection systems (IDSs) in two different ways, according to two different paradigms: anomaly detection and misuse detection. In anomaly detection systems $[17,18,10]$, historical data about a system's activity and specifications of the intended behavior of users and applications are used to build a profile of the "normal" operation of the system. The intrusion detection system then tries to identify patterns of activity that deviate from the defined profile. Misuse detection systems take a complementary approach [16, 3, 25]. Misuse detection tools are equipped with a number of attack descriptions (or "signatures") that are matched against the stream of audit data looking for evidence that the modeled attack is occurring. Misuse and anomaly detection both have advantages and disadvantages. Misuse detection systems can perform focused analysis of the audit data and they usually produce only a few false positives, but they can 
detect only those attacks that have been modeled. Anomaly detection systems have the advantage of being able to detect previously unknown attacks. This advantage is paid for in terms of the large number of false positives and the difficulty of training a system with respect to a very dynamic environment. A recent DARPA-sponsored evaluation of intrusion detection systems [20] provided interesting feedback on the characteristics of several anomaly and misuse detection systems, which confirmed the advantages and disadvantages inherent in the two approaches.

A critical feature of misuse detection systems is the description of attack signatures. Several attack languages have been proposed by the research community. However, most of the existing languages have been developed to support intrusion detection in particular domains and environments. These languages suffer from the idiosyncrasies of their operational settings and are difficult to extend to new environments. In addition, the semantic model and the abstractions provided to the signature developer are either too low-level or not clearly defined.

Our experience with intrusion detection in different domains suggested the design of a domain-independent attack description language that could be extended in a well-defined way to match different operating environments. The result of this research is STATL, which is a language to support misuse detection. STATL defines the domain-independent features of attack scenarios and also provides constructs for extending the language to describe attacks in particular domains and environments. The STATL language has been successfully used in describing both network-based and host-based attacks, and has been tailored to very different environments, e.g., Sun Microsystems' Solaris and Microsoft's Windows NT. An implementation of the runtime support for the STATL language has also been developed and a toolset of intrusion detection systems based on STATL has been implemented [35]. Even though the language was originally developed to support the development of intrusion detection systems based on the State Transition Analysis Technique (STAT) [15], STATL is general and flexible enough to be used as a common language for different misuse detection systems.

This paper presents STATL's syntax and semantics, and describes examples of its use in both network-based and host-based intrusion detection. Section 2 describes the STAT technique and our experience with intrusion detection. Section 3 presents a classification of attack languages and describes related work in this context. Section 4 presents some desirable features for attack description languages. Section 5 gives an overview of STATL. Section 6 describes the details of STATL's syntax and gives examples of its use. Section 7 discusses our experience with the language. Section 8 gives the execution semantics, and the final section draws some conclusions and outlines future work.

\section{Experience with Intrusion Detection}

The State Transition Analysis Technique (STAT) [15] was conceived in 1992 as a misuse detection method to describe computer penetrations as sequences of actions that an attacker performs to compromise the security of a computer system. A disadvantage of most signature-based approaches has been the inability to detect new attacks. The STAT approach was conceived to mitigate this disadvantage by abstracting from the details of the modeled attacks. Actions are abstracted from their native form (e.g., standard audit records or network packets) to a higher-level representation so that similar actions in a system that may have different low-level representations are mapped to a single action type. In addition, the STAT methodology supports a modeling approach that represents only those steps in an intrusion that are necessary for the effectiveness of the attack. By abstracting away from the details of a particular attack, it is possible to detect previously unknown variations of an attack or attacks that exploit similar mechanisms.

The state transition analysis technique has been used for both host-based and network-based intrusion detection. The first implementation of the host-based tool, which is called USTAT, used as input the audit records produced by Sun Microsystems' Basic Security Module (BSM) [32]. USTAT clearly demonstrated the value of the STAT approach for intrusion detection in the UNIX operating system $[13,14,26]$. However, because the original USTAT prototype was developed in an ad hoc way, a number of characteristics of this first prototype were difficult to modify or to extend to match new environments (e.g., Windows NT). The state 


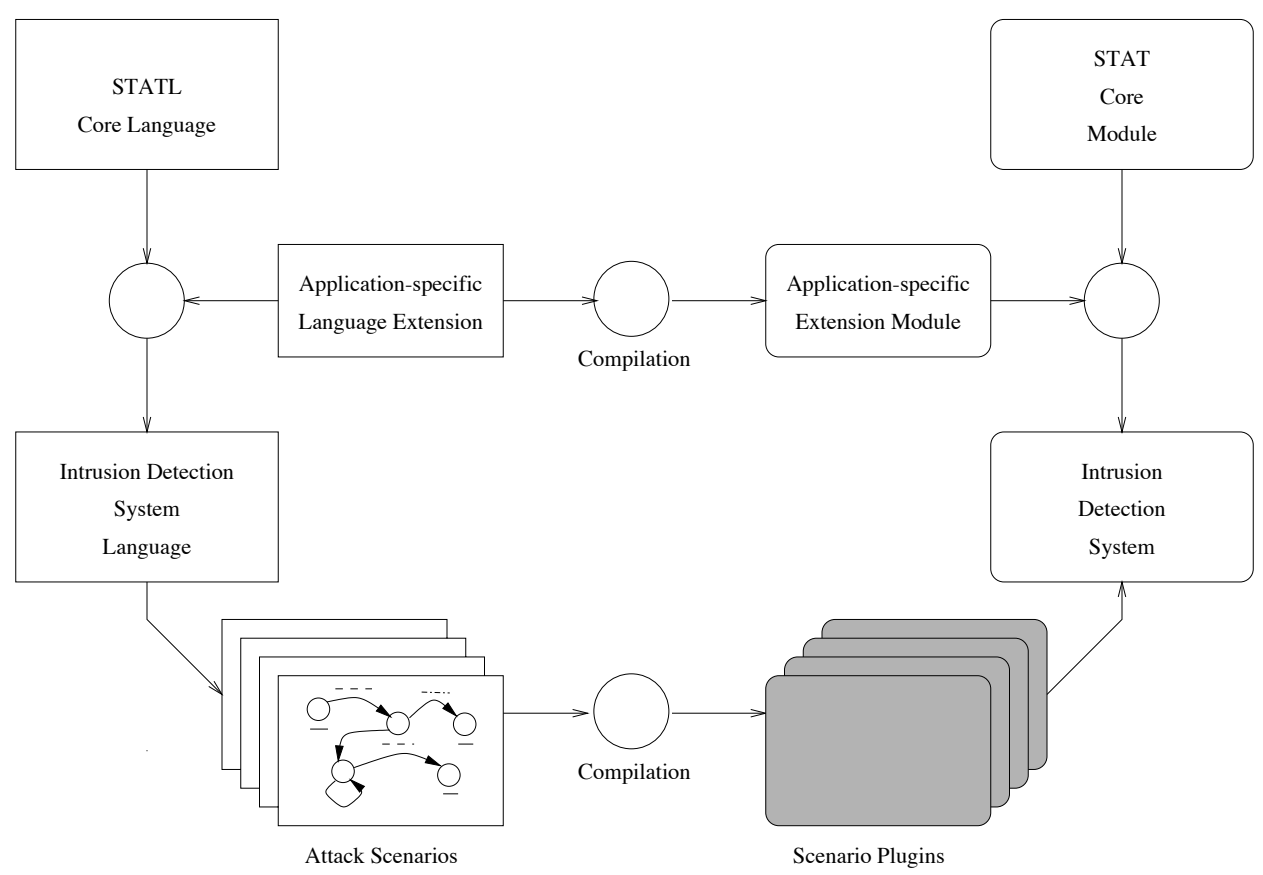

Figure 1: The STAT core module, STATL, and their extensions.

transition analysis technique was also applied to networks. The resulting tool, called NetSTAT, is aimed at realtime network-based intrusion detection [36]. The first NetSTAT prototype was also developed ad hoc, building a completely new tool that would fit the new domain.

In the original STAT model, attacks were represented using an informal state transition diagram language, with states representing snapshots of a system's security-relevant properties and resources, and transitions representing the critical signature actions of the attack. The initial USTAT implementation used a simple forward-chaining rule language, which was hard to extend, even to handle new Solaris attacks. Extending USTAT's rule language to the network domain, or even to other host operating systems, was infeasible; thus the first NetSTAT implementation represented attack signatures in C code, which was compiled and linked into the IDS. In both cases, state transition diagrams were used to develop and reason about attacks, but these diagrams were translated by hand to the form required by the target IDS.

The effectiveness of these original STAT-based tools was successfully demonstrated in the 1998 DARPA Intrusion Detection Systems Evaluation [20, 7, 8]. Participation in this evaluation revealed that although USTAT and NetSTAT were both developed in an ad hoc way and independently of each other, there were many similarities in the mechanisms they used to match attack scenarios against the input event streams. As a consequence, it was determined that the STAT-based tool suite could be redesigned as a family of systems. The toolset would share a common domain-independent language, called STATL, to represent attack scenarios, and a "core" module to support the domain-independent mechanisms used at runtime to match attack scenarios against a stream of events. The resulting STATL/core design captures the domain-independent characteristics of the STAT approach.

Because intrusion detection is performed in particular domains (e.g., hosts or networks) and in specific environments (e.g., Windows NT or Solaris), the new core-based design also provides a well-defined way to extend STATL and the core module to obtain a complete intrusion detection system, tailored to the characteristics of the specific domain and environment.

Figure 1 gives a high-level description of the roles of the core module and the STATL language in the creation of an intrusion detection system using the core-based approach. Both the STAT core module and 
the STATL core language are domain-independent. The core language is extended with domain/environmentspecific types and functions to obtain the language used to represent intrusions for a specific STAT-based tool. The language extension is compiled in the corresponding extension module, which is used with the STAT core module to construct the actual intrusion detection system. Once the STAT-based tool has been developed and the extended language defined, the intrusion detection system user (e.g., the Information Systems Security Officer) writes attack scenarios using the extended language. These attack scenarios are then compiled into dynamically linked modules, called scenario plugins. The plugin modules are dynamically loaded into the intrusion detection application at runtime, and the intrusion detection system equipped with the scenario plugins can then process the system's event stream, looking for attack signatures. The details of the compilation/translation process are given elsewhere [37, 35].

Section 7 describes the core-based STAT toolset in more detail. Developing a family of intrusion detection systems demonstrated that the core-based approach supports reuse, portability, extensibility, and customization. In addition, separating the critical domain-independent runtime mechanisms from the specific features of the different operating environments allowed for the optimization of critical functionalities. As a result, the performance of the tools increased by an order of magnitude.

\section{Attack Languages and Related Work}

Computer and network attacks are becoming more common and sophisticated and there is a need for representing, classifying, and sharing information about penetrations, exploits, and intrusion techniques. As a result, attack languages are needed to encode the "manifestations" of an attack into a suitable format, to recognize an attack given a manifestation, and to react to or report an attack. Attack languages are also useful for analyzing the relationships among different attacks in order to identify coordinated attacks against a system. In addition, attack descriptions can be used to describe attack histories/scenarios and can be used for reproducing attacks for testing purposes.

Attack languages have recently received a lot of attention from the intrusion detection community. Currently, no common language for describing attacks exists. In fact, many attacks are referred to by just one or two words, such as "buffer overflow," "SYN-flooding," or "land." Yet, it is possible to identify at least six classes of "attack languages": event languages, response languages, reporting languages, correlation languages, exploit languages, and detection languages. The different scopes and goals of each of these language classes are discussed in the following paragraphs.

Event languages are used to describe "events." These events are the basic input for security analysis. This class of languages is mainly focused on the specification of data format. In most cases, there is not a formal language definition; rather, there is some natural language description of the format of each event type and a schematic description of the associated structure. There are many examples of event languages with different formats and/or features: BSM audit record specifications [32], tcpdump packets [22], syslog messages [33], etc. A well-defined common event language would be beneficial to the intrusion detection community. It would support component and preprocessor reuse, simplify data sharing among different systems, and allow for the merging of different event streams. An example of a proposed standard format is given in [2]. Other general content specification languages, such as XML [38], could also be used.

Response languages are used to specify the actions to be taken in reaction to the detection of attack manifestations. Most IDSs currently do not have a well-defined response language. Instead they use library functions written in general-purpose languages (e.g., $\mathrm{C}$ or Java) and have limited customizability and extendibility. A common response language would be useful so that response procedures could be defined once, and installed in different intrusion detection systems. In addition, a common language would support dynamic reconfiguration of response. For example, different types of responses could be activated on the basis of the evolution of an attack.

One of the possible responses to an attack is the reporting to a human Security Officer or to an application. Reporting languages are used to describe alerts containing information about an attack, such as source of 
the attack, target of the attack, type of the attack (if known), events that are part of the manifestation, etc. Note that a reporting language could also be used as an event language at a higher level, for example as input to correlators. A common reporting language has been identified as an asset by the intrusion detection community and standard reporting language formats have been proposed. Two examples of reporting languages are the Common Intrusion Specification Language (CISL) [11] and the Intrusion Detection Message Exchange Format (IDMEF) [4]. CISL is part of the Common Intrusion Detection Framework (CIDF) [12]. The language is based on the concept of Generalized Intrusion Detection Objects, called GIDOs, which, in turn, are specified as S-expressions. IDMEF is a product of the Intrusion Detection Working Group (IDWG) of the Internet Engineering Task Force (IETF). IDMEF is based on XML and builds on much of the previous CIDF work. The language is currently undergoing the IETF standardization process.

Correlation languages are currently the focus of much research in the intrusion detection and response community. These languages rely on the semantically rich alerts provided by different intrusion detection systems and attempt to recognize "the big picture." That is, they specify relationships among attacks to identify coordinated attempts to break the security of an information system and identify the impact on the target system. The application of a correlation language is sometimes the application of existing detection techniques at a higher level of abstraction. For instance, instead of analyzing BSM events and tcpdump events, correlators may use similar techniques to analyze alerts. Examples, of current approaches to the correlation problem are the use of Bayesian networks, as demonstrated in Honeywell's ARGUS system and SRI's eBayes [34], event-based reasoning, as demonstrated in STATL, and rule-based reasoning, as in SRI's P-BEST [21].

Exploit languages are used to describe the steps to be followed to perform an intrusion. They are usually executable general-purpose languages such as $\mathrm{C}, \mathrm{C}++$, Perl, Tcl, Bourne Shell, Python, etc. There are also languages that are explicitly designed to support the scripting of attacks. Examples are CASL (Custom Attack Simulation Language) [24] and NASL (Nessus Attack Specification Language) [6]. Both these languages provide language-level support for attack scripting. A common exploit language would be useful to the security community. For example, it would simplify the generation of test cases for intrusion detection systems. In addition, it would support the sharing and understanding of attacks among the members of the security community.

Detection languages are designed to support intrusion detection, and they are usually referred to as "attack languages." These languages provide mechanisms and abstractions for identifying the manifestation of an attack. The description of an attack is in a form that can be processed by the language runtime and can be matched against a stream of input events, looking for evidence that an instance of an attack is occurring.

Examples of detection languages are N-code [28], used by the Network Flight Recorder (NFR), P-BEST [21], which is the rule-based component of SRI's Emerald, RUSSEL, which was developed as part of the ASAX project [23], Kumar's language for IDIOT, a prototype based on colored petri net representations of intrusion scenarios [19], STATL, which is used by the STAT Toolset, REE [30], the languages used by the Bro [25] and Snort [29] systems, and specification languages such as [18] and [31]. NFR, REE, Bro, and Snort are network-based IDSs. Their domain-specific languages include features that support writing concise signatures for network attacks, such as IP addresses and ordered or hashed lists with an associated membership operator. Emerald includes both host-based and network-based sensors; therefore, it needs a more general language than those used in NFR, REE, Bro, or Snort. Thus, Emerald uses a production-based expert system that includes a general-purpose rule language. RUSSEL also uses a rule-based language for audit trail analysis.

Because STATL was developed as a state/transition-based attack detection language, the remainder of this paper will concentrate on detection languages.

\section{Desirable Properties for Detection Languages}

The languages mentioned in the previous section have different characteristics that make them suitable for intrusion detection in particular environments. Although these languages proved to be useful, they lack a number of desirable properties that would support a more generalized and effective usage of the language. 
A detection language ideally provides a means of discourse about attack signatures in an abstract, IDSindependent form, but it also includes mechanized support for incorporating attack descriptions into IDSs. These detection language goals depend on the following qualities:

Simplicity: the language should provide just those features needed to represent attack scenarios.

Expressiveness: the language should be able to represent any attack signature that is detectable.

Rigor: the language should have a rigorously defined, implementation-independent syntax and semantics, so that the meaning of any attack description is unambiguous.

Extensibility: new domains have new event types, and require predicates and functions on those types. It should be possible to extend the language to new domains in a well-defined, relatively simple way.

Executability/Translatability: it should be possible to incorporate attack descriptions in an IDS application, without any manual translation. Either the specifications must be executable, or techniques and tools must be provided to translate from an attack specification to an efficient implementation.

Portability: the language processing tools should be easily adaptable to different environments.

Heterogeneity: it should be possible to describe attacks using events from multiple domains, such as IP packets and host audit records.

Evaluating detection languages with respect to the properties above is a challenging task and it is the subject of current research. In general, it is possible to note that most languages do not have a formally defined syntax and semantics. This is due partly to the fact that the languages are still in a very early stage of development and partly to the limited generality of the languages. Many languages are tailored to a particular environment and do not support a general analysis approach that can be extended to different targets. For example, Snort is widely used, but it has very limited language support and it cannot be easily extended to operate on inputs other than network packets.

STATL has been designed to provide the properties listed above. The extensibility and executability features were already described in the previous sections of this paper. The following sections focus on the language definition in terms of both syntax and semantics.

\section{STATL Overview}

A STATL specification is the description of a complete attack scenario. The attack is modeled as a sequence of steps that bring a system from an initial safe state to a final compromised state. This modeling approach is naturally supported by a state/transition-based language. One of the advantages of this approach is that state/transition specifications can be represented graphically by means of state transition diagrams (STDs). Therefore, even though STATL is primarily a text-based language, the STATL development environment includes a graphic editor that allows one to directly visualize the STD representing an attack scenario.

The STATL language provides constructs to represent an attack as a composition of states and transitions. States are used to characterize different snapshots of a system during the evolution of an attack. Obviously, it is not feasible to represent the complete state of a system (e.g., volatile memory, file system); therefore, a STATL scenario uses variables to record just those parts of the system state needed to define an attack signature (e.g., the value of a counter or the ownership of a file). A transition has an associated action that is a specification of the event that may cause the scenario to move to a new state. For example, an action may be the opening of a TCP connection or the execution of an application. The space of possible relevant actions is constrained by a transition assertion, which is a filter condition on events that may possibly match the action. For example, an assertion may require that a TCP connection is opened with a specific destination port or that an application being executed should be part of a predefined set of security-critical applications. 
It is possible that several occurrences of the same attack are active at the same time. A STATL attack scenario therefore has an operational semantics in terms of a set of instances of the same scenario prototype. The scenario prototype represents the scenario's definition and global environment, and the scenario instances represent attacks currently in progress.

The evolution of the set of instances of a scenario is determined by the type of transitions in the scenario definition. A transition can be consuming, nonconsuming, or unwinding. A nonconsuming transition is used to represent a step of an occurring attack that does not prevent further occurrences of attacks from spawning from the transition's source state. Therefore, when a nonconsuming transition fires, the source state remains valid, and the destination state becomes valid too. For example, if an attack has two steps that are the creation of a link named " $-i$ " to a SUID shell script and the execution of the script through the created link, then the second step does not invalidate the previous state. That is, another execution of the script through the same link may occur. Semantically, the firing of a nonconsuming transition causes the creation of a new instance. The original instance is still in the original state, while the new instance is in the state that is the destination state of the fired transition. In contrast, the firing of a consuming transition makes the source state of a particular attack occurrence invalid. Semantically, the firing of a consuming transition does not generate a new scenario instance; it simply changes the state of the original one. Unwinding transitions represent a form of "rollback" and they are used to describe events and conditions that may invalidate the progress of one or more scenario instances and require the return to an earlier state. For example, the deletion of a file may invalidate a condition needed for an attack to complete, and, therefore, the corresponding scenario instances may be brought back to a previous state, such as before the file was created. The details of scenario execution are presented in Section 8.

\section{STATL Syntax, Informal Semantics, and Examples}

As discussed in Section 4, an attack detection language should have a rigorously defined syntax and semantics. This section presents STATL's syntax and informal semantics. It also includes fragmentary examples for each of the syntax rules. In the syntax rules, literal keywords are in boldface and other literal text is enclosed in single quotes. Optional items are enclosed in square brackets '[', ']', items that may appear zero or more times are enclosed in curly braces ' $\{$ ', ' $\}$ '. Alternatives are separated by 'l' and grouped with parentheses where necessary to indicate associativity. Examples may include ellipses (...) to indicate that details have been left out; the ellipses are not part of STATL.

\subsection{Lexical Elements}

STATL identifiers consist of letters, digits, and the underscore character ' 2 , , and start with a letter. For example host_name and IPaddr 2 are identifiers. STATL identifiers are case-sensitive, so IPaddress is different from IPAddress. STATL compound identifiers use standard object-oriented dot notation, as in "object.attribute". STATL keywords are reserved words and may not be used as identifiers. For example, since scenario is a keyword, it may not be used as a variable name.

STATL includes two kinds of comments: any text between "/*” and "*/" (except “*/"), including the delimiters, is a comment. Any text following "//" to the end of the line, including the "// " marker, is a comment. Whitespace may appear anywhere in a STATL specification except within tokens (keywords, identifiers, and multiple-character operators).

\subsection{Data Types}

STATL includes several built-in types: int and $u$ int in various sizes, bool, string, timeval (for timestamps), and timer (described in Section 6.10). It also includes arrays, plus containers vector, set, list, and map. It is not possible to define new data types within a STATL scenario. Application-specific types must be defined within the application-specific extension library. For example, NetSTAT scenarios may use different types than USTAT scenarios, but both use int and timeval. 


\subsection{Scenario}

A scenario uses zero or more libraries of application-specific types, events, functions, and predicates. A scenario has a name, may have parameters, may contain annotations and constant and variable declarations, and most importantly, contains the states and transitions that define the "attack signature" - what to match and what to do with matches. A scenario may also define supporting functions to be used in state and transition assertions and code blocks:

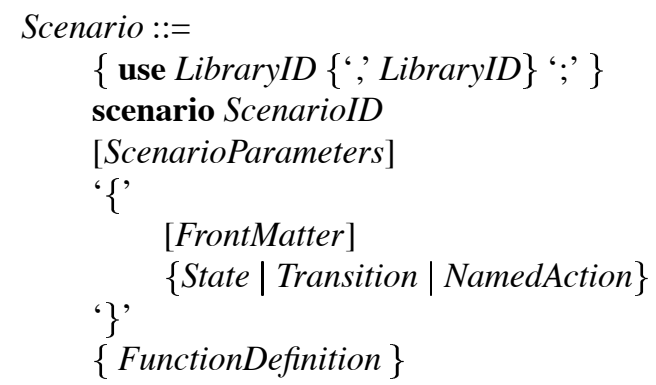

A scenario must have at least one transition and two states - the initial state and a final state. The initial state must have no incoming transitions, and final states have no outgoing transitions. Scenario parameters are specified as a list of comma-separated typed identifiers:

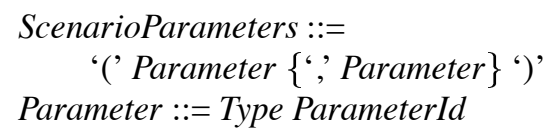

Example:

scenario example (string host, int count) $\{\ldots\}$

The example scenario has two parameters, host and count. Parameters are accessible by the scenario instances as global constants.

\subsection{Front Matter}

Scenarios may include annotations, and may declare constants and variables:

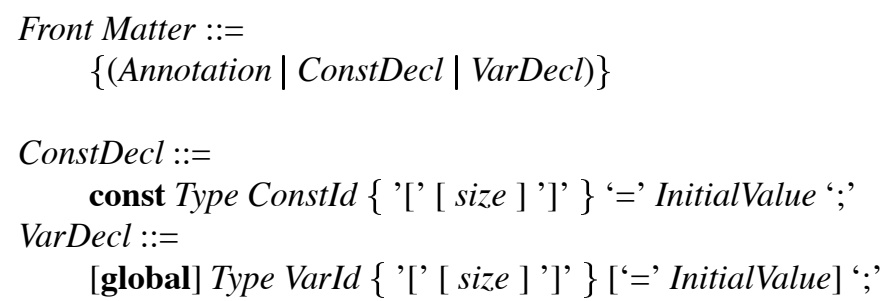

Annotations are described in Section 6.12. A variable declared "global" is shared by all instances of the scenario. A variable not declared "global" is instantiated privately in each instance of the scenario. Variables may be assigned initial values.

Example: 


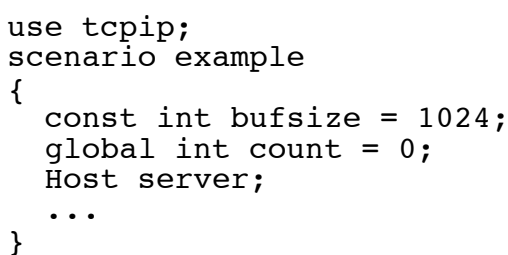

This example declares a constant integer bufsize with value 1024 and declares a global variable count with initial value 0 . This variable will be shared by all instances of the scenario. That is, if a scenario instance increments the count variable, the update is seen by all other instances of the scenario. The variable declaration in the example also includes a variable named server of type Host (a tcpiplib type). Because server is a local variable (i.e., its declaration does not contain the keyword global), each instance of the scenario will have its own copy of server.

\subsection{State}

"State" is one of the two fundamental concepts in STATL. States have names so they can be referred to in transitions and in the graphical representation of the scenario (i.e., in the STD). Each state may have annotations, an assertion, and a code block, but these elements are optional:

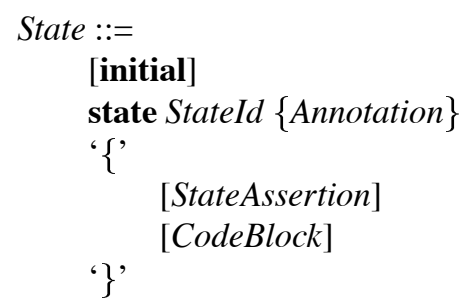

Exactly one state must be designated as the initial state. When a scenario plugin is loaded into an IDS a first instance is created in the initial state.

The state assertion, if present, is tested before entry to the state, after testing the assertion of the transition that leads to the state. A state's assertion is implicitly True if none is specified. A state's code block is executed after the incoming transition's assertion and the state's assertion have been evaluated and found to be True, and after the incoming transition's code block (if it exists) is executed.

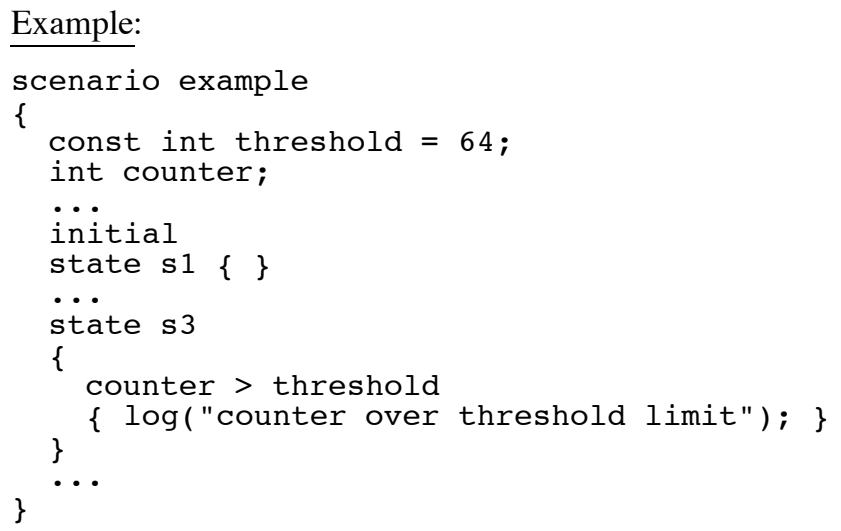

In this example state $\mathbf{s} 1$ is designated as the initial state. It has neither an assertion nor a code block. State $\mathbf{s} 3$ has an assertion and a code block. The assertion specifies that the value of local variable counter is greater than the value of constant threshold. The code block calls the built-in procedure log to write a message to the IDS's log file. 


\subsection{Transition}

"Transition" is the second of the two fundamental concepts in STATL. Each transition has a name and must indicate the pair of states that it connects. Transitions may have the same source and destination state; that is, loops are allowed. In addition, a transition may have annotations, must specify a type, must specify an event type to match, and may have a code block:

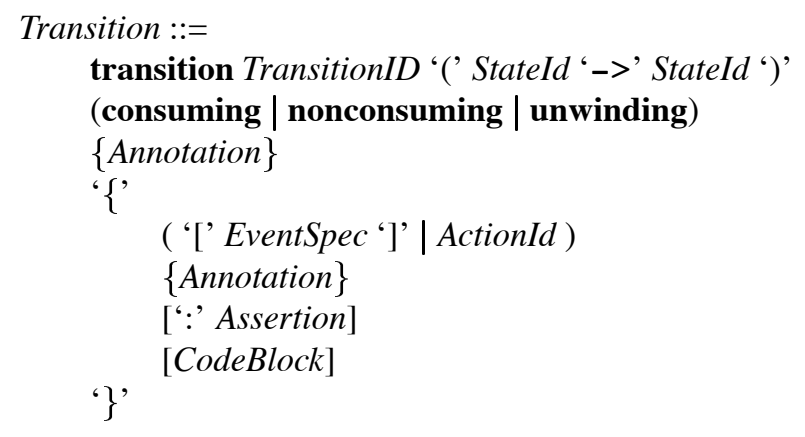

A transition's event is specified either directly (see Section 6.7) or by reference to a named signature action (see Section 6.8). In the former case the transition's assertion is just the assertion in the transition. In the latter case, if the named signature action includes an assertion and the transition also includes an assertion, then the resulting assertion is the conjunction of the two assertions. An example is given in Section 6.8, after named signature actions are defined.

A transition's code block is executed after evaluating the transition's assertion and the destination state's assertion, and before executing the destination state's code block. More precisely, the order of evaluation of assertions and the execution of code blocks, after matching an event type (defined in Section 6.7), is as follows:

1. evaluate the transition assertion. If True, then

2. evaluate the state assertion. If True, then

3. execute the transition code block, possibly modifying local and global environments, and then

4. execute the state code block, possibly modifying local and global environments. ${ }^{1}$

Transitions are deterministic, which means that every enabled transition fires if its assertion and the destination state's assertion are satisfied. A transition's code block may perform any computation supported by STATL and the IDS extension in use, but is typically used to copy event field values into the global or local environment for later reference.

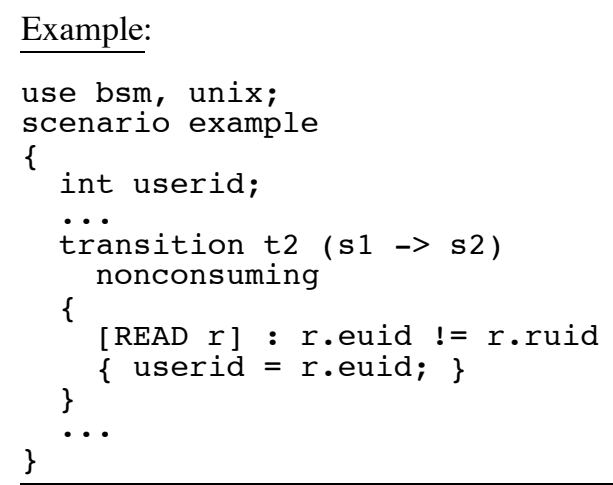

\footnotetext{
${ }^{1}$ An alternative would be to execute the transition codeblock before evaluating the state assertion. However, this would require backtracking to undo environment changes when the state assertion is not satisfied. Otherwise the environment could be changed for "partially" fired transitions, which would be semantically unsatisfactory.
} 
In this example, $t 2$ is a nonconsuming transition that leads from state $\mathbf{s} 1$ to state $\mathrm{s} 2$. The event spec indicates that the transition should match events of type READ, with a filter condition specifying that the euid and ruid fields of the event must differ for the transition to fire. The transition's code block copies the euid field of event $r$ into the local variable userid for later reference. Note that this scenario uses both bsmlib and unixlib extensions, which define BSM events and UNIX-related abstractions, respectively.

\subsection{EventSpec}

"Event specs" are the essential elements of transitions. They specify what events (signature actions) to match and under what conditions.

EventSpec ::=

( BasicEventSpec [SubEventSpec] )|TimerEvent

BasicEventSpec ::= EventType EventId

SubEventSpec ::= '[' EventSpec $\{$ ', EventSpec $\}$ ']'

EventType ::= ANY | ApplEventType '(' ApplEventType \{'l'ApplEventType $\}$ ')'

An event spec is either a basic event spec optionally followed by a subevent spec, or it is a timer event (see Section 6.10). A basic event spec identifies the built-in meta-event "type" ANY, which matches any event, or an application-specific event type (e.g., READ) or a disjunction of application-specific event types (e.g., (UDP | TCP ) ), and a name that will be used to reference the matching event. A basic event spec identifying a single type matches an event of the same type only. A basic event spec that is the disjunction of two or more event types matches an event of any of the types in the disjunction. A subevent spec identifies a set of event specs. Subevent specs enable complex, tree-structured event patterns. A subevent spec matches a set of subevents if each event spec in the subevent spec matches one of the events in the set.

Example:

$\left[\begin{array}{r|l}\text { (READ } \mid \text { WRITE ) access ] : } \\ \text { access. euid } !=\text { a }\end{array}\right.$

access.euid != access.ruid

Example:

[ IP d1 [TCP t1] ] :

$(\mathrm{d} 1 . \operatorname{src}==192.168 .0 .1) \& \&(\mathrm{t} 1 . \mathrm{dst}==23)$

The first example is a USTAT event spec that matches read or write events in which the effective and real userids differ. The second example is a NetSTAT event spec (with a subevent spec) that matches any IP datagram containing a TCP segment, with source IP address 192.168.0.1 and destination port 23.

The built-in meta-event type ANY is effectively the same as disjunction over all application-specific event types, but is easier to specify (and more efficient to implement as a special case).

\subsection{NamedSigAction}

A named signature action has a name and specifies an event spec, and may have annotations:

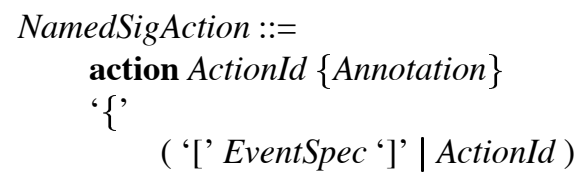




\section{$\{$ Annotation $\}$ \\ ['?'Assertion] \\ '}

Named signature actions may be used to improve clarity and maintainability when multiple transitions have identical or similar actions; for example, having the same action type but slightly different assertions. In such cases the common part can be factored out, put into a named signature action, and then used in the similar transitions.

Example:

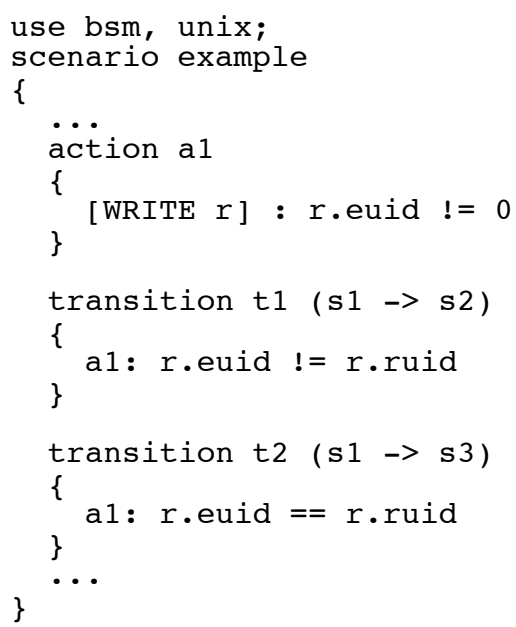

In this example transitions $t 1$ and $t 2$ both use named signature action $a 1$ as their event spec, but with different assertions. This is equivalent to:

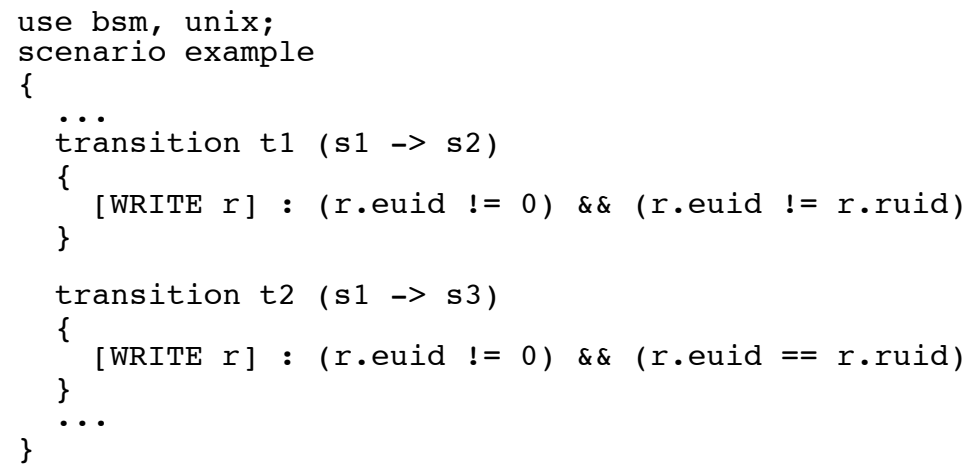

\subsection{CodeBlock}

Transitions and states may have code blocks that are executed after the corresponding transition and state assertions have been evaluated and found to be True. A code block is a sequence of statements enclosed in braces:

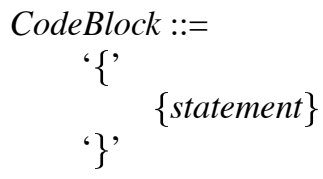


The statements in a codeblock can be assignments, for and while loops, if-then-else, procedure calls, etc. Semantically, the statements in a STATL code block are executed in order, in the context of the global and local environments of the scenario instance in which the code block is executed.

\subsection{Timers}

Timers are useful to express attacks in which some event or set of events must (or must not) happen within an interval following some other event or set of events. Timers can also be used to prevent "zombie" scenarios scenarios that have no possible evolution - from wasting memory resources.

Timers are declared as variables using the built-in type timer. There are both local and global timers. All timers must be explicitly declared. Timers are started in code blocks using the built-in procedure timer start. Timer expiration is treated as an event, and these events may be matched by using "timer events" as transition event specs.

\section{Example:}

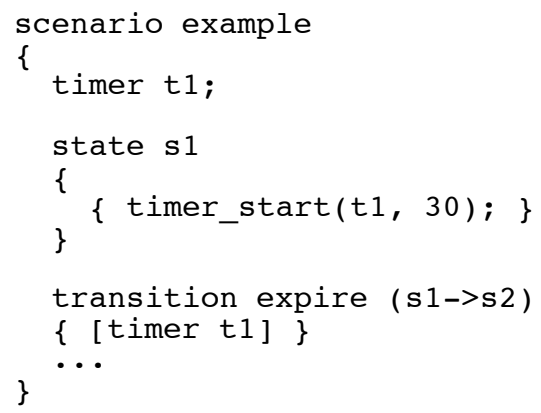

The code block of state $\mathbf{s} 1$ starts timer $t 1$, which will expire in 30 seconds (i.e., at a time 30 seconds later than the timestamp on the event that led to state $\mathbf{s} 1)$. The timer event timer $t 1$ matches the expiration of the timer named $t 1$. When timer $t 1$ expires, transition expire will fire, leading to state $s 2$.

Starting a timer that is already "running" resets that timer. A single timer may appear in multiple transitions; every enabled transition that has timer $t$ as its event spec fires when the timer expires.

\subsection{Assertions}

Assertions appear as filter conditions in states and in event specs (which are the matching element of transitions). STATL assertions are built up from literal constants, variable and constant names, function calls, and common arithmetic and relational operators. A STATL assertion is evaluated at runtime in the context of the global and local environments of the scenario instance where it is evaluated.

Assertions may use, but may not change, the value of any name in the global or local environment. In addition, transition assertions may refer to the events named in the event spec and to the fields of those events.

\subsection{Annotations}

STATL annotations provide a standard way to extend the language with application-specific features. The annotations to a scenario are processed by a specialized component of the offline STAT architecture, called the Analyzer [37]. The Analyzer's task is to perform any IDS-specific preprocessing on a STATL scenario before the scenario is actually translated into an executable representation. For example, the NetSTAT Analyzer uses the annotations of a STATL scenario to determine where a probe should be placed in a target network, and how the probe should be configured in order to be sure to detect the described attack. The Analyzer relies on another 
component, the Factbase, to obtain information about the target environment. For example, in NetSTAT the Factbase contains information about the network topology and the services deployed.

Syntactically, an annotation is an annotation tag optionally followed by a list of expressions, all enclosed in the annotation delimiters " $<*$ " and "* $>$ ":

Annotation ::=

<*Id $\{$ Expression $\{$ ', Expression $\}$ *>

Annotations may be attached to states, transitions, event specs, named signature actions, and scenarios. Each IDS defines any scenario customization it needs as annotations, and the associated Analyzer is expected to process them (any annotations left by the Analyzer will be ignored by the STATL translator).

An example of an application-specific language extension is the ENDPOINT PORTS annotation, often used in NetSTAT actions to specify which two network interfaces are communicating. For example, the event spec:

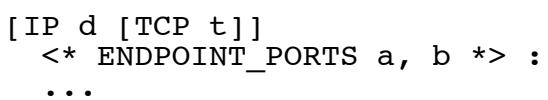

represents a TCP/IP segment exchanged between network interfaces a and $\mathrm{b}$.

Annotations are attached to states, transitions, and named signature actions by putting them just before the entity's opening brace. For example,

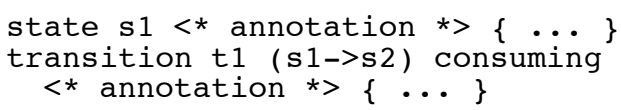

An annotation in the scenario front matter is at scenario scope. An example of this kind is the set of conditions in NetSTAT scenarios that customize the attack signature to an installation-specific Factbase. These are specified using the NetSTAT CONSTRAINT annotation:

Example:

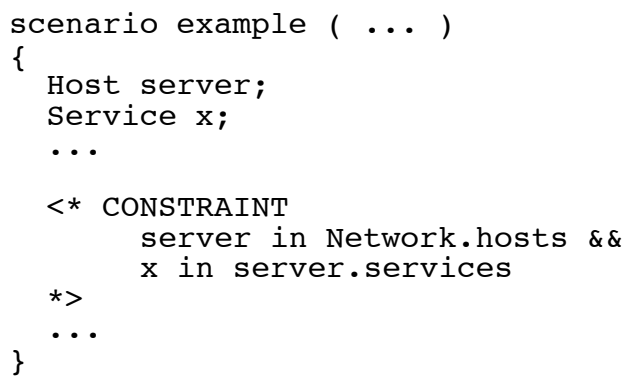

In this example the annotation tag is CONSTRAINT and the argument list consists of one expression.

\section{Experience with the Language}

The STAT core-based framework, which is centered around STATL and the STAT core component, has been used to generate a new tool suite based on the STAT approach. The tool suite includes USTAT, WinSTAT, and NetSTAT. The core-based STAT tool suite was recently used in the 1999 DARPA Intrusion Detection Evaluation effort. For each tool in the suite an extension module containing the application-specific abstract event types and predicates was developed. In addition, an application-specific preprocessor was developed to translate the native audit records into abstract event representations. For each tool a Factbase and Analyzer were designed to translate STATL scenarios into executable plugins. 
USTAT is the core-based implementation of the original Unix host-based IDS. It uses Sun Microsystems' BSM as a source of audit data. USTAT relies on two STATL extension libraries called bsmlib and unixlib. The USTAT preprocessor filters the incoming audit records and translates the selected records into USTAT events, which are defined as an abstraction of the original audit records in a BSM-specific extension module. USTAT uses only 35 of the 125 different audit record types generated by BSM, and those 35 are translated into 20 USTAT event types. The USTAT Factbase is composed of a number of filesets that characterize the security aspects of critical files and applications in the host's filesystem. For example, the "restricted-write-directories" fileset specifies a list of directories in which non-root users should not make changes (e.g., /bin, /usr/lib). Each fileset has one or more associated scenarios that define the meaning of the fileset. For example, associated with the "restricted-write-directories" fileset is a scenario that raises an alarm if any create, write, or delete action by a non-root user succeeds in one of the listed directories. USTAT's Analyzer makes various changes to scenarios to implement application-specific semantics that are not part of the core. For example, if a USTAT scenario instance depends on the existence of a process, then the instance can be unwound when that process exits. The USTAT Analyzer implements this by adding unwinding transitions to the original scenario as needed.

WinSTAT is a new tool that performs host-based intrusion detection in the Windows NT environment. WinSTAT uses the event logs produced by Windows NT as input and transforms a selection of NT events into WinSTAT events. The WinSTAT Factbase and Analyzer are very similar to those for USTAT.

NetSTAT is a network-based intrusion detection system. The core-based approach has been used to develop the NetSTAT probe component. A NetSTAT probe uses the network traffic sniffed from a network segment as input. It can also work offline by reading tcpdump files. NetSTAT relies on a STATL extension library called tcpiplib, which includes 22 event types and more than 50 predicates and functions. The NetSTAT preprocessor is responsible for the filtering of relevant network packets and for the parsing and abstracting of network events. The NetSTAT preprocessor tasks include reassembling fragmented IP datagrams, reassembling TCP streams, parsing DNS and RPC events, and maintaining relevant information about the state of the network (e.g., active connections). The NetSTAT Factbase component is a repository that stores and manages the security relevant information about a network, such as the network topology and the network services deployed. The Analyzer uses the annotations in STATL scenarios to determine where the probes must be placed in the protected network and how the probes must be configured [37].

STATL has been used as a basis to develop scenarios for USTAT, WinSTAT, and NetSTAT. In the following sections examples of actual STATL scenarios are given for USTAT and NetSTAT. The attacks presented are well-known simple examples. The intent is to allow the reader to concentrate on understanding the language, rather than the attack itself.

\subsection{USTAT Example}

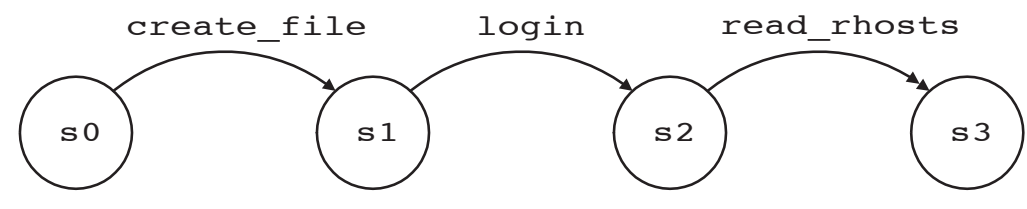

Figure 2: ftp-write state transition diagram.

In the $f t p$-write attack, an attacker uses the $f t p$ service to create a bogus . rhosts file in a world-writable $f t p$ daemon home directory. Using the created file the attacker is then able to open a remote session using the rlogin service without being required to supply a password. A generalization of this attack is that an attacker creates a bogus .rhosts file in any other user's home directory and then uses it to be allowed to login without providing a password. This generalization of the ftp-write attack is depicted schematically in the STD in Figure 2. The different types of arrows are used to denote different types of transitions in STDs: a solid arc with a single arrowhead denotes a nonconsuming transition, a solid arc with a double arrowhead denotes a 
consuming transition, and a dashed arc denotes an unwinding transition. A text-based STATL specification of the generalized attack follows.

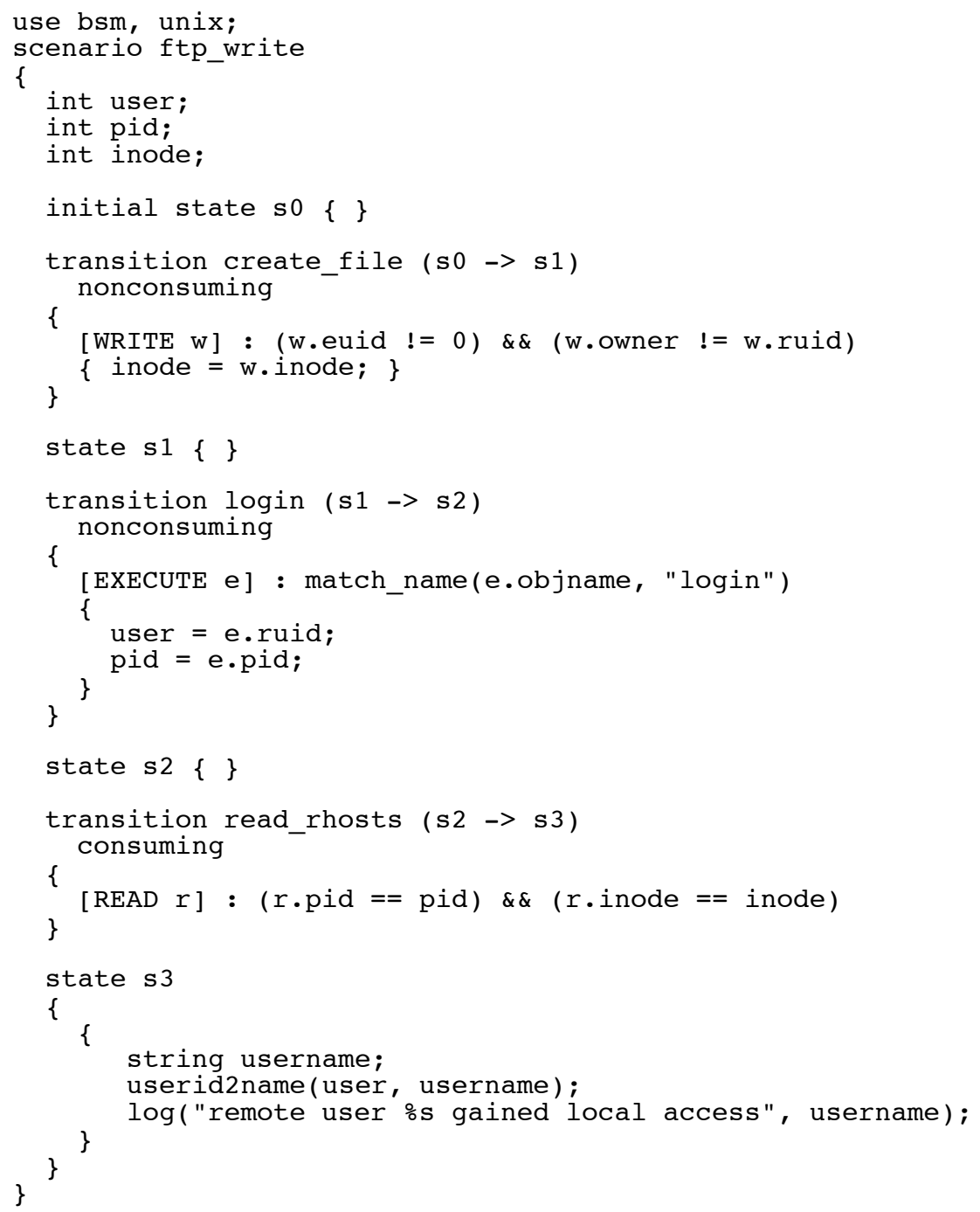

The sequence of events detected by this scenario is that a file is created (or written to) by a non-root user who doesn't own the directory containing the file, and then the login program runs and reads the suspicious file. WRITE, EXECUTE, and READ are abstractions of BSM-specific event types. The predicate match name() and procedure userid2name() are part of the UNIX extension. This signature was successfully used during the 1999 DARPA Intrusion Detection Evaluation, with the addition of three unwinding transitions, as shown in the STD in Figure 3:

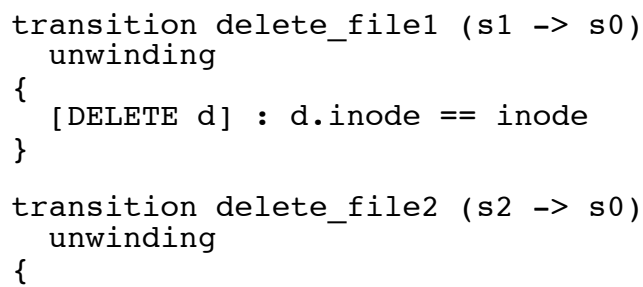




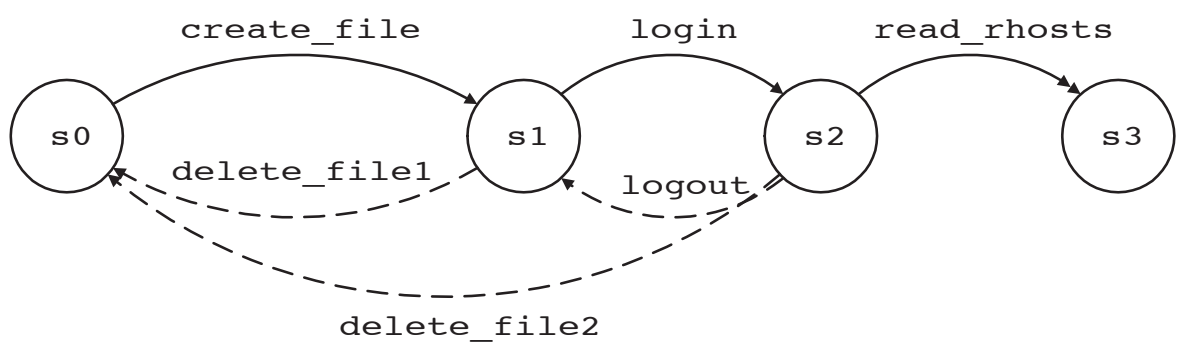

Figure 3: ftp-write state transition diagram with unwinding transitions.

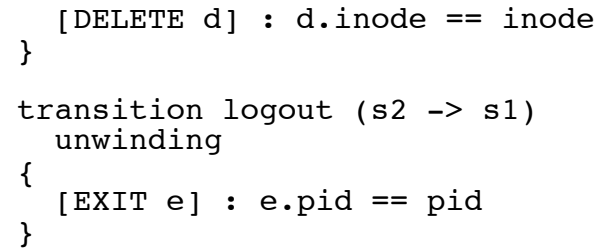

The delete_file transitions roll the attack back to the initial state, because no further progress can be made after the suspicious file is deleted. The logout transition rolls the attack back to state $\mathbf{s} 1$, because after the login process exits, it obviously cannot read any files.

\subsection{NetSTAT Example}

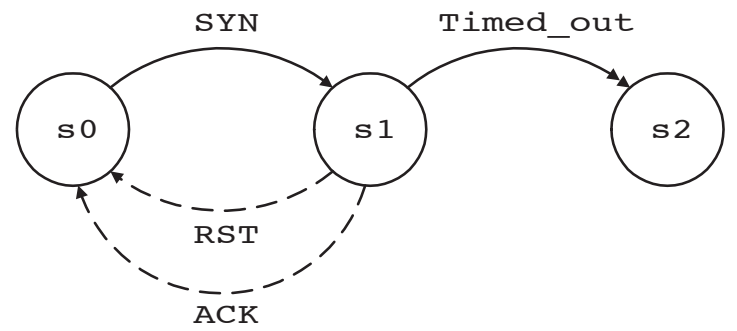

Figure 4: Half-Open TCP state transition diagram.

This example scenario detects half-open TCP connections. A TCP connection is in a half-open state when the three-way handshake of the TCP protocol is not completed and the server is waiting for the final acknowledgment from the client [27]. This attack is the building block for the SYN-flooding denial of service attack [5]. The goal of the SYN-flooding attack is to consume all the server-side resources dedicated to the setup of TCP connections, effectively preventing the server from accepting any other TCP connection. The STD for the hal fopen scenario is shown in Figure 4. The STATL specification for this scenario follows.

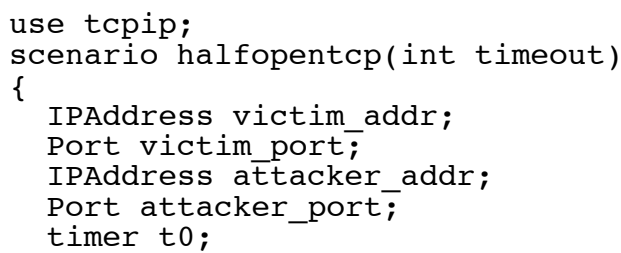




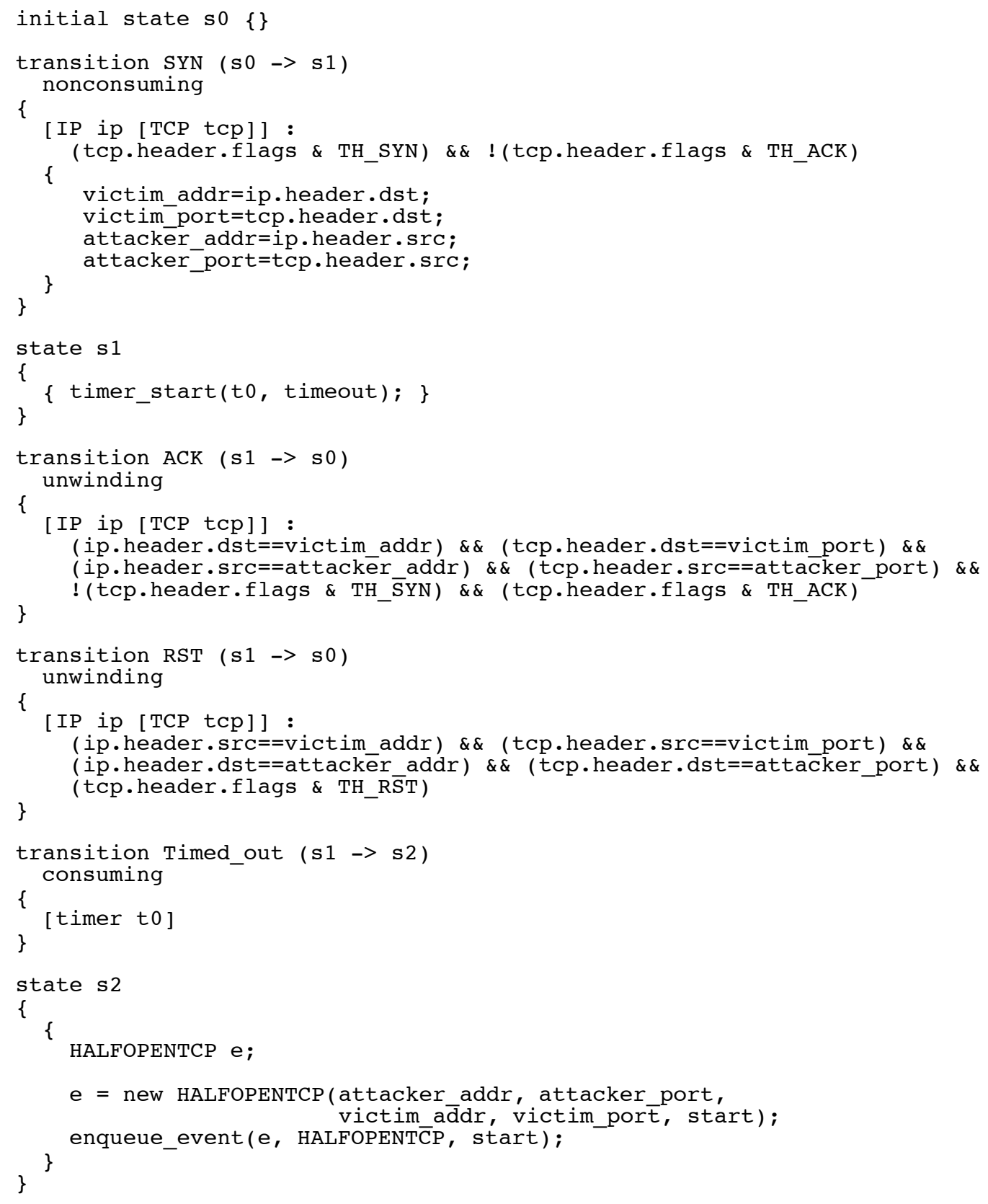

In the halfopentcp scenario the attacker sends a TCP SYN segment asking for the creation of a TCP connection. This event fires transition SYN. In the transition code block, the IP addresses and TCP ports involved are saved to local variables. Transition SYN leads to state $\mathbf{s} 1$ and a timer is started using the scenario parameter timeout for the timeout value.

In state s 1 two events may invalidate the attack. Firstly, the server may answer with an RST segment shutting down the connection and freeing the resources dedicated to the establishment of the TCP connection. Secondly, the client may send the final ACK of the TCP setup hand-shake and switch the connection to an established state. These two events fire transitions RST and ACK, respectively. The two transitions are both unwinding and bring the scenario to its initial state, meaning that this instance of the scenario does not correspond 
to an attack in progress.

If the handshake is not completed by the client and the server does not answer with an RST message, then the timer will fire and transition Timed_out will bring the scenario to state s2. In this state a new event of type HALFOPENTCP is created on the basis of the information gathered in the previous steps of the scenario. The event is encapsulated in a generic STAT event and sent to the language runtime. This event, called a synthetic event, is inserted in the network event stream and may be processed by other scenarios (e.g., a scenario to detect the SYN-flooding attack). Synthetic events enable forward chaining between different scenarios.

\section{Scenario Execution Semantics}

STATL is centered around the concept of attack scenarios. An attack scenario has a set of static elements and a dynamic behavior. The static elements of a scenario are states and transitions, as introduced in Section 6. The dynamic behavior of a scenario defines how a scenario evolves when it is actually matched against a stream of input events. An informal description of the matching process and of the corresponding scenario evolution has been given in the previous sections of the paper. In this section a more formal description of the semantics of scenario execution is given. A formal definition for the complete language is given in [9].

A STATL scenario is defined formally as a 6-tuple:

$$
\mathrm{S}:\left(S, T, s_{0}, I, g, q\right)
$$

where $S$ is a set of states, $T$ is a set of transitions, $s_{0}$ identifies the initial state, $I$ is an instance set, $g$ is a global environment, and $q$ is a global timer queue. State, transition, and initial state are the same as introduced in Section 6. The global environment $g \in \mathcal{G}$ is a name-value mapping for scenario global variables, which are also called "scenario variables". The names in a global environment are the scenario's declared global variables, parameters, and constants. The global timer queue $q \in \mathcal{Q}$ contains timeout events that apply to all instances of a scenario.

An instance set $I$ is a set of instances:

$$
I: \wp(\mathcal{I})
$$

Each instance $i \in \mathcal{I}$ is a non-empty sequence of snapshots:

$$
i: \Sigma^{+}
$$

A snapshot $\sigma \in \Sigma$ contains a state name, a local environment, a local timer queue, and an event:

$$
\sigma: \mathcal{N}_{S} \times \mathcal{L} \times \mathcal{Q} \times \mathrm{E}
$$

where $\mathcal{N}_{S}$ denotes the domain of state names, $\mathcal{L}$ is the domain of local environments, and $\mathrm{E}$ is the domain of STAT events. The local environment is a name-value mapping like the global environment. The only difference is that a local environment is associated with a single instance while a global environment is shared among the possibly many instances of a single scenario. Similarly, a local timer queue contains timeout events that apply to a single instance.

Intuitively, a snapshot $\sigma$ identifies how far an attack has progressed by recording the current state, the values of local variables, and the event that led to the current state. The state, local environment, and event, of snapshot $\sigma$ are defined by $\sigma$.state, $\sigma$.env, and $\sigma$.event, respectively. The current state $\sigma$.state implicitly identifies which transitions are enabled (i.e., available to match events) in snapshot $\sigma$. The enabled transitions in a set of transitions $T$ with respect to snapshot $\sigma$ are those whose source state is the same as $\sigma$.state:

$$
\operatorname{enabled}(T, \sigma)=\left\{t_{j} \in T \ni t_{j} \text {.from }=\sigma . \text { state }\right\}
$$


where ' $\ni$ ' means "such that"

By representing a scenario instance $i$ as a sequence of snapshots $\left\langle\sigma_{0} \ldots \sigma_{n}\right\rangle$, it is possible to represent the complete history of an attack that (partially) matches a particular scenario S. Each snapshot in an instance, other than the first, corresponds to a transition that fired in reaction to a particular event. The enabled transitions in a set of transitions $T$ with respect to instance $i$ are determined by the last snapshot of $i$, denoted by $i$.last:

$$
\operatorname{enabled}(T, i)=\operatorname{enabled}(T, i . \text { last })=\left\{t_{j} \in T \ni t_{j} \text {.from }=i \text {.last.state }\right\}
$$

Intuitively, the instance set $I=\left\{i_{0}, i_{1}, \ldots, i_{m}\right\}$ records all partial matches of $\mathrm{S}$ up to the "current" event. That is, $I$ contains all the active instances of a particular attack. There is no notion of an enabled transition set with respect to an instance set $I$. Instead, each instance $i \in I$ has an enabled transition set.

A scenario starts from an initial safe state and reaches a final state through transitions. Every scenario S, initially has:

$$
\mathrm{S} . I=\left\{i_{0}\right\}, \text { where } i_{0}=\left\langle\sigma_{0}\right\rangle \text { and } \sigma_{0}=\left(\mathrm{S} . s_{0}, l_{0},\langle\rangle \text {, null }\right)
$$

That is, every instance set $I$ initially contains a single instance, which, in turn, consists of a single initial snapshot. The initial snapshot $\sigma_{0}$ contains the initial state $s_{0}$ as defined for scenario $\mathrm{S}$, a local environment $l_{0}$ in which each local variable has its initial value, an empty timer queue, and no event. In this initial state, the enabled transitions are the enabled transitions of $i_{0}$, which are just the transitions that exit the scenario's initial state $s_{0}$ :

$$
\operatorname{enabled}\left(T, i_{0}\right)=\operatorname{enabled}\left(T, i_{0} . \text { last }\right)=\operatorname{enabled}\left(T, \sigma_{0}\right)=\left\{t_{j} \in T \ni t_{j} . \text { from }=s_{0}\right\}
$$

Scenario instances evolve as a consequence of event matching. E denotes the domain of STAT events. Events are not part of the syntax of STATL, but are needed to define the semantics of STATL. Therefore, one speaks of "STAT events", not "STATL events", and "event" is used to mean "STAT event" rather than "application event". Each STAT event $\epsilon \in$ E contains an application event type, a timestamp, an application event, and a possibly empty set of subevents, each of which is a STAT event of the same form. The formal definition of STAT events is given elsewhere [9]. In the following, a STAT event is considered to be an entity that can be matched against the event spec and assertion of a transition.

Given a scenario $\mathrm{S}=\left(S, T, s_{0}, I, g, q\right)$ and a current event $\epsilon, \mathrm{S}$ evolves to $\left(S, T, s_{0}, I^{\prime}, g^{\prime}, q^{\prime}\right)$ according to the algorithm in Figure 5. The auxiliary set $F$ of to-be-fired transition-instance pairs is initially empty, as is the new instance set $I^{\prime}$. The new global environment $g^{\prime}$ and the new global timer queue $q^{\prime}$ are initially the same as the old $g$ and $q$, respectively.

Informally, the algorithm says to first identify all the transitions that should fire, along with the instances in which they should fire, and the event that causes each transition to fire. Next initialize $I^{\prime}$ to the set of instances in $I$ that have no transitions to be fired. Then fire any nonconsuming transitions that were identified in the first step. After that, fire the identified consuming transitions. Finally, fire the identified unwinding transitions. The following subsections define the functions fire _* $\operatorname{transition}\left(t, i, \epsilon, I^{\prime}, g^{\prime}, q^{\prime}\right)$ by specifying how $I^{\prime}, g^{\prime}$, and $q^{\prime}$ are changed. In each case it is assumed that the instance $i$ in which the transition is firing is of the form $i=\left\langle\sigma_{0} \ldots \sigma_{n}\right\rangle$. The functions eval_trans_assertion and eval_state assertion, which evaluate the state and transition assertions, and the function matching events, which performs the matching of events against event specs, are described elsewhere [9].

\subsection{Nonconsuming Transitions}

Informally, when a nonconsuming transition is fired, a copy of the instance is created and a new snapshot is appended to the copy. The state of the snapshot is the destination state of the transition that fired. It has as its event the event that caused the transition to fire. And it has as its local environment the local environment of the original instance, updated by executing the transition codeblock and the destination state codeblock. The 


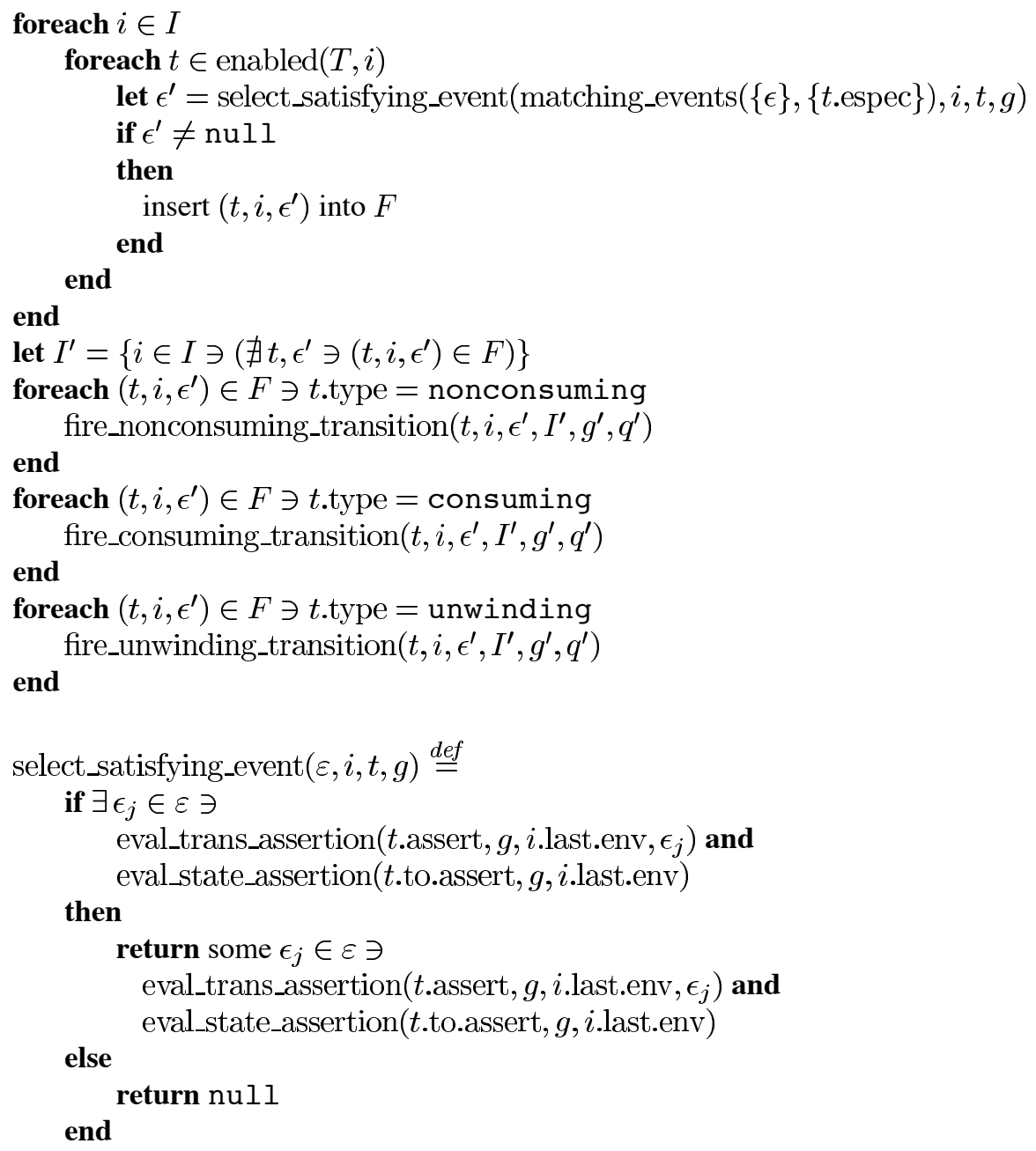

Figure 5: STATL event processing algorithm

original instance is added to the new instance set. If the destination state is not a final state, the new instance is also added to the new instance set. A final state is a state $s$ with no exiting transitions; that is, one for which $\left\{t_{j} \in T \ni t_{j}\right.$.from $\left.=s\right\}=\emptyset$.

The function fire_nonconsuming_transition $\left(t, i, \epsilon, I^{\prime}, g^{\prime}, q^{\prime}\right)$ is defined as follows:

$$
\begin{aligned}
& I^{\prime}= \begin{cases}I^{\prime} \cup\left\{i,\left\langle\sigma_{0} \ldots \sigma_{n} \sigma_{n+1}\right\rangle\right\} & \text { if } t . \text { to is not final } \\
I^{\prime} \cup\{i\} & \text { otherwise }\end{cases} \\
& g^{\prime}=\operatorname{exec}\left(t \cdot \text { to.code, null, } \operatorname{exec}\left(t \cdot \operatorname{code}, \epsilon,\left(\left(g^{\prime}, \sigma_{n} \text {.env }\right),\left(q^{\prime}, \sigma_{n} \cdot q\right)\right)\right)\right) \text {.maps.first } \\
& q^{\prime}=\operatorname{exec}\left(t \text {.to.code, null, } \operatorname{exec}\left(t \cdot \operatorname{code}, \epsilon,\left(\left(g^{\prime}, \sigma_{n} . \operatorname{env}\right),\left(q^{\prime}, \sigma_{n} \cdot q\right)\right)\right)\right) \text {.queues.first }
\end{aligned}
$$

where $\sigma_{n+1}$ is constructed as follows:

$$
\sigma_{n+1} . \text { state }=t . t o
$$




$$
\begin{aligned}
\sigma_{n+1} . \text { env } & =\operatorname{exec}\left(t \cdot \text { to.code, null, } \operatorname{exec}\left(t \cdot \operatorname{code}, \epsilon,\left(\left(g^{\prime}, \sigma_{n} . \text { env }\right),\left(q^{\prime}, \sigma_{n} \cdot q\right)\right)\right)\right) \cdot \text { maps.second } \\
\sigma_{n+1} \cdot \mathrm{q} & =\operatorname{exec}\left(t \cdot \text { to.code, null, } \operatorname{exec}\left(t \cdot \operatorname{code}, \epsilon,\left(\left(g^{\prime}, \sigma_{n} . \text { env }\right),\left(q^{\prime}, \sigma_{n} \cdot \mathrm{q}\right)\right)\right)\right) \text {.queues.second } \\
\sigma_{n+1} \cdot \text { event } & =\epsilon
\end{aligned}
$$

The original and new instances are added to the new instance set $I^{\prime}$. The global and local environments and timer queues are updated by (1) executing the transition codeblock in the context of the current event, the local environment from the original instance, and the current global environment and global timer queue, then (2) executing the state codeblock in the context of no event, and the name-value mappings produced by executing the transition codeblock.

\subsection{Consuming Transitions}

When a consuming transition is fired, a copy of the instance is created and a new snapshot is appended to the copy. The new snapshot is constructed as in the case of a nonconsuming transition. If the destination state is not a final state, the new instance is added to the new instance set. The original instance is not added to the new instance set.

The function fire_consuming_transition $\left(t, i, \epsilon, I^{\prime}, g^{\prime}, q^{\prime}\right)$ is defined as follows. The new global environment $g^{\prime}$ and new global timer queue $q^{\prime}$ are defined as for nonconsuming transitions. The new instance set $I^{\prime}$ is defined as follows:

$$
I^{\prime}= \begin{cases}I^{\prime} \cup\left\{\left\langle\sigma_{0} \ldots \sigma_{n} \sigma_{n+1}\right\rangle\right\} & \text { if } t . \text { to is not final } \\ I^{\prime} & \text { otherwise }\end{cases}
$$

where $\sigma_{n+1}$ is constructed as for nonconsuming transitions. The only difference between a consuming transition and a nonconsuming transition is that when a consuming transition is fired, the original instance is not added to the new instance set.

\subsection{Unwinding Transitions}

When an unwinding transition fires, one or more instances are deleted from the instance set (i.e., they are not in the new instance set). The instances that get deleted are just those that are derived from the snapshot that is being unwound to.

The function fire_unwinding_transition $\left(t, i, \epsilon, I^{\prime}, g^{\prime}, q^{\prime}\right)$ is defined as follows. The new global environment $g^{\prime}$ and new global timer queue $q$ are defined as for consuming and nonconsuming transitions. To derive $I^{\prime}$, it is necessary to define

$$
p=\sigma_{0} \sigma_{1} \ldots \sigma_{j} \sigma_{j+1}, j<n
$$

where $\sigma_{j}$ is the first snapshot in $i$ with $\sigma_{j}$.state $=t$. to. $^{2} I^{\prime}$ is then defined as follows:

$$
I^{\prime}=\left(I^{\prime} \backslash\left\{i^{\prime} \in I^{\prime} \ni p \in i^{\prime}\right\}\right) \cup\left\{\left\langle\sigma_{0} \ldots \sigma_{j}\right\rangle\right\}
$$

This says to delete from $I^{\prime}$ any instance $i^{\prime}$ that has $p$ as a prefix, because any such instance must be derived from the instance that is being unwound and must have progressed past the state (snapshot) that is being unwound to. Then add to $I^{\prime}$ the instance $\left\langle\sigma_{0} \ldots \sigma_{j}\right\rangle$, which is the instance that is being unwound to. Notice that instances are being deleted from $I^{\prime}$, not from $I$, and this is done after all nonconsuming and consuming transitions have fired.

\footnotetext{
${ }^{2}$ The definition could have specified "last" instead of "first", or it could have been left to the scenario writer.
} 


\section{Conclusions and Future Work}

The STATL language has been designed to meet the requirements for an attack description language as described in Section 3. The language is simple and explicitly focused on representing attack scenarios. The resulting language includes a few well-defined abstractions (scenario, state, transition, action) that are used to specify attack signatures. The language is expressive. Currently, 18 attack scenarios have been developed for USTAT, 10 attacks have been developed for WinSTAT, and more than 20 attacks have been coded for the NetSTAT tool. In encoding attacks no limit in the expressiveness of the language was found. Different types of attacks were coded: probes, denial-of-service, remote and local user-to-root attacks, threshold-based attacks, window-based attacks, and even policy-based attacks. In addition, the modeled attacks differ in the level of abstraction. Some attacks exploit weaknesses in the TCP/IP protocol stack and are low level, while others can be abstracted and detect a wide number of variations (for example all the local buffer overflow attacks considered so far are detected by a single USTAT/STATL scenario). The language is rigorous, that is, it has a precise syntax and a parser for the language. The semantics of the language has been formally defined and an executor for the language has been developed. STATL is also extensible. Describing attacks in both the network and host domains demonstrated that very different types of events and predicates could be easily developed and integrated into the language. The language extensibility also allows for the management of heterogenous data; that is, by composing different language extensions it is possible to develop a STATL extended language that is able to manage events from different audit streams (e.g., network packets and host audit records). Finally, STATL is portable. The tools used to develop scenarios and process the resulting executable representations have been developed in portable languages like Java and Tcl, and by using a development environment (the GNU Build system), which allows for easy porting to new platforms.

The first prototype of the STATL development environment is almost complete. The translator tool has been implemented, but the application-specific Analyzers have not, so some of the customization and configuration is currently done manually. The language extension process uses an object-oriented framework supporting the creation of IDS extensions, making it possible to automate some of the tasks involved in developing event types and predicates for new environments and domains.

To prove the general applicability of STATL to state-based intrusion detection, an analysis of the possible translation between STATL and other attack languages is being conducted. For example, Snort [29] and NFR [28] have been investigated, and STATL subsets that are translatable to each have been identified. Additional attack languages (e.g., P-BEST [21]) will be analyzed in the future, and prototype translators will be developed for some.

Other future work will be focused on the translation process and on the improvement of the features of the STATL language. In addition, attack signatures will be collected and new STATL scenarios and STATL extensions will be developed.

\section{Acknowledgments}

This research was supported by the Defense Advanced Research Projects Agency (DARPA) and Rome Laboratory, Air Force Materiel Command, USAF, under agreement number F30602-97-1-0207, and by the National Security Agency's University Research Program, under agreement number MDA904-98-C-A891. The U.S. Government is authorized to reproduce and distribute reprints for Governmental purposes notwithstanding any copyright annotation thereon.

The views and conclusions contained herein are those of the authors and should not be interpreted as necessarily representing the official policies or endorsements, either expressed or implied, of the Defense Advanced Research Projects Agency (DARPA), Rome Laboratory, the National Security Agency, or the U.S. Government. 


\section{References}

[1] J.P. Anderson. Computer Security Threat Monitoring and Surveillance. James P. Anderson Co., Fort Washington, April 1980.

[2] M. Bishop. Standard Audit Trail Format. In Proceedings of the 1995 National Information Systems Security Conference, pages 136-145, Baltimore, Maryland, October 1995.

[3] CISCO. NetRanger Intrusion Detection System. Technical Information, April 1999.

[4] D. Curry. Intrusion Detection Message Exchange Format: Extensible Markup Language (XML) Document Type Definition. draft-ietf-idwg-idmef-xml-01.txt, July 2000.

[5] daemon9. Project neptune. Phrack Magazine, 7(48), July 1996.

[6] R. Deraison. The Nessus Attack Scripting Language Reference Guide, 2000. http: //www. nessus.org.

[7] R. Durst, T. Champion, B. Witten, E. Miller, and L. Spagnuolo. Addendum to "Testing and Evaluating Computer Intrusion Detection Systems”. CACM, 42(9):15, September 1999.

[8] R. Durst, T. Champion, B. Witten, E. Miller, and L. Spagnuolo. Testing and Evaluating Computer Intrusion Detection Systems. CACM, 42(7):53-61, July 1999.

[9] S. Eckmann, G. Vigna, and R. Kemmerer. STATL Definition. Technical Report TRCS00-19, Department of Computer Science, UC Santa Barbara, September 2000.

[10] A.K. Ghosh, J. Wanken, and F. Charron. Detecting Anomalous and Unknown Intrusions Against Programs. In Proceedings of the Annual Computer Security Application Conference (ACSAC'98), Scottsdale, AZ, December 1998.

[11] Common Intrusion Detection Framework Working Group. A CISL Tutorial. http://www.gidos.org/tutorial.html, 2000.

[12] Common Intrusion Detection Framework Working Group. Common Intrusion Detection Framework Specification. http://www.gidos.org/, 2000.

[13] K. Ilgun. USTAT: A Real-time Intrusion Detection System for UNIX. Master's thesis, Computer Science Department, University of California, Santa Barbara, July 1992.

[14] K. Ilgun. USTAT: A Real-time Intrusion Detection System for UNIX. In Proceedings of the IEEE Symposium on Research on Security and Privacy, Oakland, CA, May 1993.

[15] K. Ilgun, R.A. Kemmerer, and P.A. Porras. State Transition Analysis: A Rule-Based Intrusion Detection System. IEEE Transactions on Software Engineering, 21(3), March 1995.

[16] Internet Security Systems. Introduction to RealSecure Version 3.0, January 1999.

[17] H. S. Javitz and A. Valdes. The NIDES Statistical Component Description and Justification. Technical report, SRI International, Menlo Park, CA, March 1994.

[18] C. Ko, M. Ruschitzka, and K. Levitt. Execution Monitoring of Security-Critical Programs in Distributed Systems: A Specification-based Approach. In Proceedings of the 1997 IEEE Symposium on Security and Privacy, pages 175-187, 1997.

[19] S. Kumar. Classification and Detection of Computer Intrusions. PhD thesis, Purdue University, 1995. 
[20] MIT Lincoln Laboratory. DARPA Intrusion Detection Evaluation. http://www.ll.mit.edu/IST/ideval/, 1999.

[21] U.Lindqvist and P.A. Porras. Detecting Computer and Network Misuse with the Production-Based Expert System Toolset (P-BEST). In IEEE Symposium on Security and Privacy, Oakland, California, May 1999.

[22] S. McCanne, C. Leres, and V. Jacobson. Tcpdump 3.4. Documentation, 1998.

[23] A. Mounji. Languages and Tools for Rule-Based Distributed Intrusion Detection. PhD thesis, Facultés Universitaires Notre-Dame de la Paix Namur (Belgium), September 1997.

[24] Secure Networks. Custom Attack Simulation Language (CASL), January 1998.

[25] V. Paxson. Bro: A System for Detecting Network Intruders in Real-Time. In Proceedings of the 7th USENIX Security Symposium, San Antonio, TX, January 1998.

[26] P.A. Porras. STAT - A State Transition Analysis Tool for Intrusion Detection. Master's thesis, Computer Science Department, University of California, Santa Barbara, June 1992.

[27] J. Postel. Transmission Control Protocol. RFC 793, September 1981.

[28] M.J. Ranum, K. Landfield, M. Stolarchuck, M. Sienkiewicz, A. Lambeth, and E. Wall. Implementing a Generalized Tool for Network Monitoring. In Eleventh Systems Administration Conference (LISA '97). USENIX, October 1997.

[29] M. Roesch. Writing Snort Rules: How To write Snort rules and keep your sanity. http: //www. snort.org.

[30] R. Sekar, V. Guang, S. Verma, and T. Shanbhag. A High-performance Network Intrusion Detection System. In Proceedings of the 6th ACM Conference on Computer and Communications Security, November 1999.

[31] R. Sekar and P. Uppuluri. Synthesizing Fast Intrusion Detection/Prevention Systems from High-Level Specifications. In Proceedings of the USENIX Security Symposium, 1999.

[32] Sun Microsystems, Inc. Installing, Administering, and Using the Basic Security Module. 2550 Garcia Ave., Mountain View, CA 94043, December 1991.

[33] syslog(3). UNIX documentation.

[34] A. Valdes and K. Skinner. An Approach to Sensor Correlation. In Proceedings of RAID 2000, Tolouse, France, October 2000.

[35] G. Vigna, S. Eckmann, and R. Kemmerer. The STAT Tool Suite. In Proceedings of DISCEX 2000, Hilton Head, South Carolina, January 2000. IEEE Computer Society Press.

[36] G. Vigna and R.A. Kemmerer. NetSTAT: A Network-based Intrusion Detection Approach. In Proceedings of the $14^{\text {th }}$ Annual Computer Security Application Conference, Scottsdale, Arizona, December 1998.

[37] G. Vigna and R.A. Kemmerer. NetSTAT: A Network-based Intrusion Detection System. Journal of Computer Security, 7(1):37-71, 1999.

[38] World Wide Web Consortium (W3C). Extensible Markup Language (XML). W3C Recommendation, February 1998. http://www.w3.org/TR/1998/REC-xml-19980210. 


\section{Figures}

This is not a real paper section. It contains copies of figures that are also "inlined" in the body of the paper.

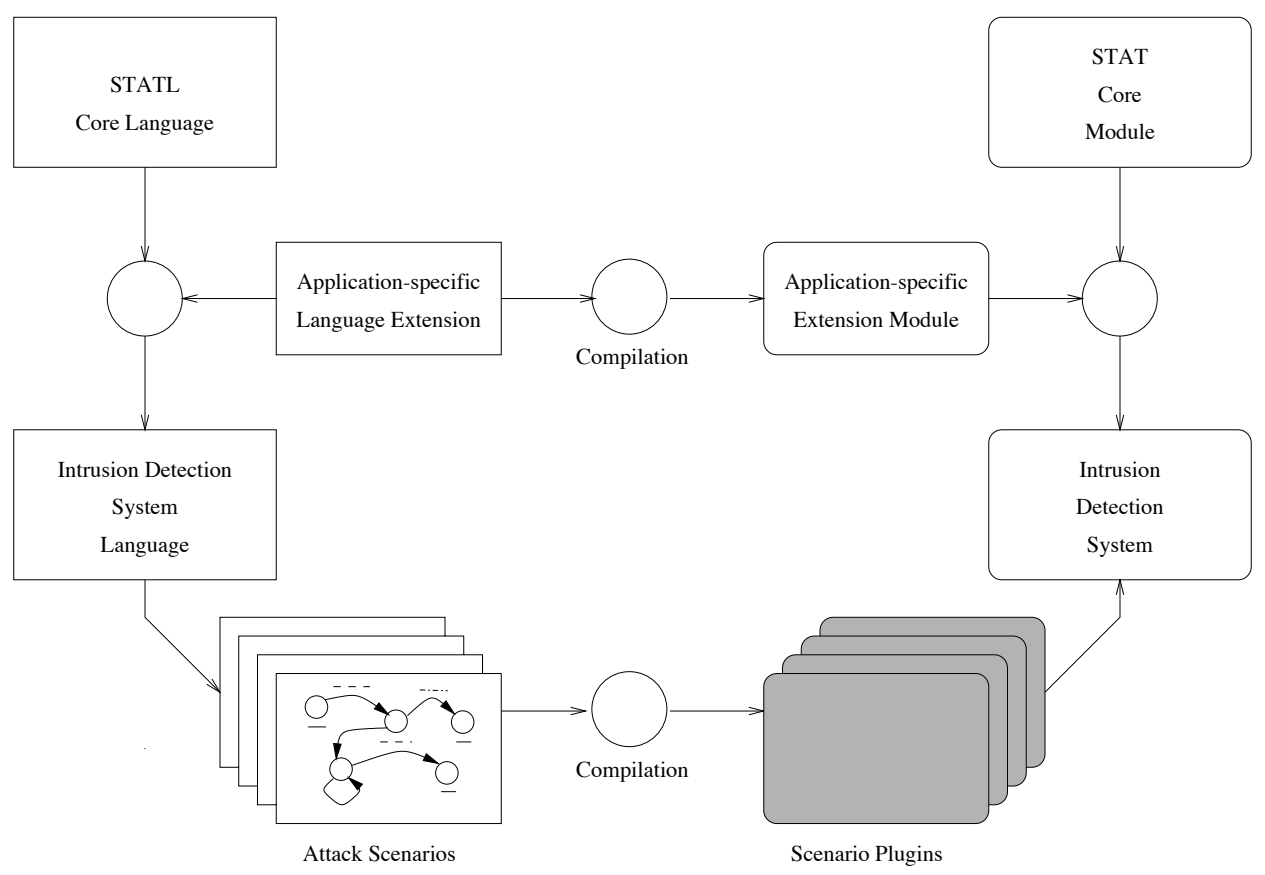

Figure 6: The STAT core module, STATL, and their extensions. 


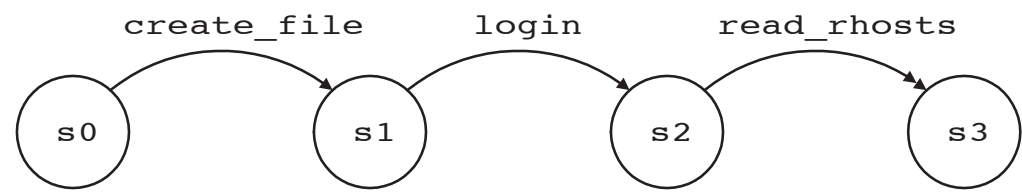

Figure 7: ftp-write state transition diagram. 


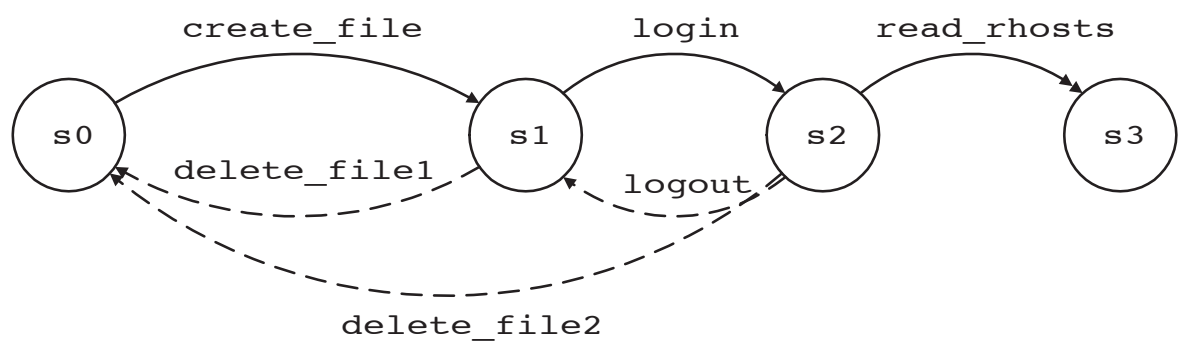

Figure 8: ftp-write state transition diagram with unwinding transitions. 


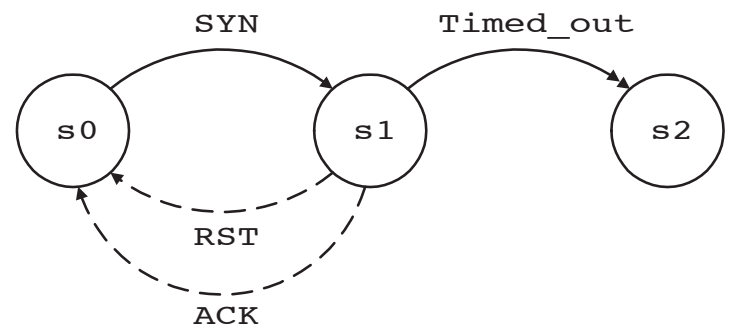

Figure 9: Half-Open TCP state transition diagram. 